\title{
Upper airway mucosa temperature in obstructive sleep apnoea/ hypopnoea syndrome, nonapnoeic snorers and nonsnorers
}

\author{
F. Sériès, I. Marc
}

Upper airway mucosa temperature in obstructive sleep apnoea/hypopnoea syndrome, nonapnoeic snorers and nonsnorers. F. Sériès, I. Marc. (OERS Journals Ltd 1998.

ABSTRACT: Upper airway (UA) inflammation (oedema and hyperaemia) is frequently observed in snorers and patients with sleep apnoea/hypopnoea patients.

The temperature of different UA regions measured in 11 male nonsnorers, 13 nonapnoeic snorers and 10 untreated sleep apnoea/hypopnoea syndrome (SAHS) patients using infra-red video recording. Measurements were taken with the mouth open during tidal volume mouth breathing, and at the beginning and end of a $10 \mathrm{~s}$ end-inspiratory voluntary apnoea which followed either mouth or nasal breathing. Three measurements were obtained from the uvula and from each side of the posterior pharyngeal wall and two from each side of the soft palate. The different UA regions were characterized by their inspiratory temperature, the expiratory rewarming and the changes in UA temperature during apnoea.

The temperature of the uvula was significantly lower than that of the other UA regions. For each anatomical region, there were no differences in inspiratory temperature between normals, snorers and SAHS. In normals, the expiratory rewarming was significantly higher in the uvula than in the velum and the posterior pharyngeal wall, whereas these regional differences were not observed in snorers and SAHS. The velum and posterior pharyngeal rewarming was significantly less in normals than in snorers and SAHS. During apnoea, the UA rewarming was similar to that observed during expiration.

In conclusion, tissue temperature varies between the different upper airway regions and during tidal breathing and the intensity of the regional expiratory rewarming differs between normals, snorers and patients with sleep apnoea/hypopnoea syndrome. Eur Respir J 1998; 12: 193-197.
Unité de Recherche, Centre de Pneumologie, Hôpital Laval, Université Laval Québec, Canada.

Correspondence: F. Sériès

Centre de Pneumologie

Hôpital Laval

2725 Chemin Sainte-Foy

Sainte-Foy

Québec G1V 4G5

Canada

Fax: 14186564762

Keywords: Infra-red thermography sleep apnoea syndrome

upper airway inflammation

Received: August 261997

Accepted after revision March 111998

Supported by the Respiratory Health Network of Centres of Excellence of Canada.
The sleep apnoea/hypopnoea syndrome (SAHS) is characterized by the occurrence of sleep-induced repetitive episodes of complete or incomplete upper airway (UA) closure. The UA collapses occur mainly at the nasopharyngeal level, but can involve the oropharyngeal and hypopharyngeal structures [1]. During nonapnoeic episodes, as during snoring, the UA behaves as a Starling resistor [2, 3], resulting in a fluttering of the uvula, soft palate, pharyngeal walls and tongue with increasing pharyngeal transmural pressure gradient. The UA closes when the pharyngeal pressure decreases below a critical pressure level. During apnoeic events, the activity of UA dilator muscles progressively increases, the maximal activity being reached with electroencephalographic (EEG) arousal [4]. This results in UA reopening when the dilating forces counterbalance the suction forces.

The pressure required to restore UA patency (opening pressure) is significantly higher than the closing pressure, presumably owing to the adherent properties of the mucosal surface [5]. This suggests that in both nonapnoeic snorers and SAHS, recurrent injuries to the UA tissues may occur owing to the vibration of UA structures associated with UA fluttering. In SAHS, this tissue injury could also be accounted for by the mechanical effects of UA tissue stretching, resulting from the dilating and collapsing forces applied on the UA. This could contribute to the development of the trauma of pharyngeal tissues, as suggested by the histopathological differences observed in the uvula and soft palate between apnoeic and nonapnoeic snorers [6], and potentially to UA inflammation with local oedema and hyperaemia. However, to the authors' knowledge, regional differences in UA inflammation between normals and patients with breathing abnormalities during sleep have not been specifically investigated.

Infra-red thermography has been widely used in medicine to document the presence of and to quantify inflammation in cutaneous [7] and subcutaneous tissues such as breasts [8], and joints [9], but also in mucosal oral surfaces $[10,11]$. Furthermore, the rewarming rate of the cooled gingival mucosa has been shown to increase with increasing inflammation [11]. It was hypothesized that UA mucosa tissue temperature and rewarming characteristics should be modified by nocturnal obstructive breathing abnormalities and a study was designed using infra-red thermography to compare the temperature of different UA regions in normal nonsnorers, nonapnoeic snorers, and SAHS. 


\section{Materials and methods}

\section{Patients}

Thirty four subjects participated in the study. These included 11 male nonsnorers, 13 nonapnoeic snorers ( 12 male, 1 female) and 10 untreated SAHS (9 male, 1 female). All were nonsmokers for at least $1 \mathrm{yr}$ and were free of allergy and UA infection. They were not receiving any medication. Their nocturnal pulmonary status was clinically assessed and confirmed by a conventional sleep study (see below).

\section{Sleep studies}

Polysomnographic studies consisted of continuous acquisition of measurements from an electroencephalogram $\left(\mathrm{C}_{4} / \mathrm{A}_{1}, \mathrm{C}_{3} / \mathrm{A}_{2}, \mathrm{O}_{2} / \mathrm{A}_{1}\right.$ and $\left.\mathrm{O}_{1} / \mathrm{A}_{2}\right)$, a submental electromyogram, an electro-oculogram and an electrocardiogram, nasobuccal airflow with thermistors, thoraco-abdominal movements by inductive plethysmography (Respitrace, Ambulatory Monitoring Inc, Arsdley, NY, USA), arterial oxyhaemoglobin saturation $\left(\mathrm{Sa}_{\mathrm{a}} \mathrm{O}_{2}\right)$ with an ear oximeter (504 Pulse oximeter, Criticare Systems, Waukesha, WI, USA) and breathing noises with two microphones placed at the head of the bed [12]. All variables were recorded on computer (Sandman, Melleville Diagnostics, Ottawa, Ontario, Canada).

\section{Study design}

Temperature recordings were made during the month following the sleep recording. Subjects sat in front of an infra-red camera (Inframetrics, model 600, Billerica, MA, USA) placed approximately $30 \mathrm{~cm}$ in front of the mouth. A video recording of the UA (mouth opened) was obtained on three different occasions: during tidal mouth breathing, during a $10 \mathrm{~s}$ end-inspiratory voluntary apnoea following mouth breathing and during a $10 \mathrm{~s}$ end-inspiratory voluntary apnoea following nasal breathing. During tidal breathing, inspiratory and expiratory time were measured for each respiratory cycle. The temperature and humidity of the room in which measurements were taken remained constant during the study period. The protocol was authorized by the Institutional Research Board and written informed consent was obtained from each subject.

\section{Data collection and statistical analysis}

Sleep studies. Polysomnographic recordings were manually interpreted in $30 \mathrm{~s}$ epochs. Sleep parameters were determined according to RECHTSCHAFFEN and KALES criteria [13]. Arousals were scored according to the American Sleep Disorders Association (ASDA) definition [14] and the number of arousals per hour of sleep was determined. Apnoea was defined as the cessation of nasal-oral airflow for at least $10 \mathrm{~s}$. Hypopnoea was defined as a $>50 \%$ decrease in the sum of thoracoabdominal movements for at least $10 \mathrm{~s}$ associated with a $2 \%$ drop in $\mathrm{Sa}_{2} \mathrm{O}_{2}$ and/or an arousal.
Temperature measurements. Following data collection, temperature measurements were obtained with dedicated computer software (Thermogram 3.0, Thermotechnix Systems, Cambridge, UK) that determines the temperature at a spot level of $\sim 1 \mathrm{~mm}^{2}$ which can be located anywhere on the freeze-frame video image with a $0.1{ }^{\circ} \mathrm{C}$ sensitivity [15]. During the tidal breathing trial, end-inspiratory and end-expiratory measurements were obtained. During the apnoeic trials, measurements were taken at the beginning and at the end of the apnoea. The different areas at which measurements were taken are identified by different letters in figure 1 . Three measurements were obtained on the uvula: at the margin of the soft palate (a), at the tip of the uvula (b) and midway between the two (c). Six posterior pharyngeal wall measurements were taken on each side of the uvula, two were done at the horizontal level of point $b$ (laterally close to the palatal arch (d) and midway between the palatal arch and the uvula (e)) and one at the horizontal of point $c$ above point e (f). Four soft palate measurements were obtained on each side of the uvula above spots $\mathrm{d}$ and $\mathrm{f}(\mathrm{g}, \mathrm{h})$. These anatomical spots were retained because data were available during the different procedures and in each subject of the different groups. The different values obtained at each anatomical region were averaged to characterize the local temperature during tidal breathing and apnoea.

Comparisons between groups for each anatomical region and inspiratory-expiratory time and between anatomical regions within each group were performed with an analysis of variance (ANOVA) followed by a StudentNewman-Keul's test for multiple comparison. Regression analysis between UA mucosa temperature and nocturnal breathing characteristics was evaluated by Spearman rank correlation. Statistical significance was inferred for comparisons in which $\mathrm{p}<0.05$.

\section{Results}

Anthropometric characteristics of the different groups are presented in table 1. As expected, UA temperatures

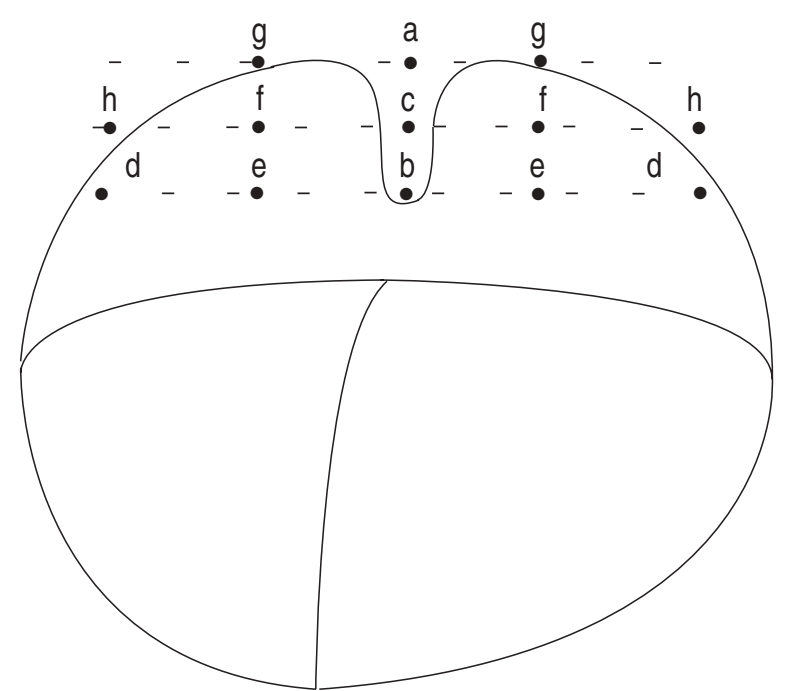

Fig. 1. - Schematic representation of the different spots where upper airway temperature measurements were obtained. Three measurements were obtained on the uvula $(\mathrm{a}-\mathrm{c})$, three others on each side of the posterior pharyngeal wall $(\mathrm{d}-\mathrm{f})$ and two on each side of the soft palate $(\mathrm{g}, \mathrm{h})$. 
Table 1. - Anthropomorphic and nocturnal breathing characteristics of the participating subjects

\begin{tabular}{|c|c|c|c|}
\hline & $\begin{array}{c}\text { Normals } \\
n=11\end{array}$ & $\begin{array}{c}\text { Snorers } \\
n=13\end{array}$ & $\begin{array}{c}\text { SAHS } \\
n=10\end{array}$ \\
\hline Age yrs & $40 \pm 8^{a}$ & $43 \pm 10^{a}$ & $49 \pm 9 a$ \\
\hline $\mathrm{BMI} \mathrm{kg} \cdot \mathrm{m}^{-2}$ & $24 \pm 2^{a}$ & $29 \pm 5^{\mathrm{ab}}$ & $32 \pm 6^{b}$ \\
\hline $\begin{array}{l}\text { Snoring index } \\
\mathrm{n} \cdot \mathrm{h}^{-1}\end{array}$ & $29 \pm 19$ a & $213 \pm 112^{b}$ & - \\
\hline AHI $n \cdot h^{-1}$ & $2.4 \pm 2.0^{\mathrm{a}}$ & $9.0 \pm 6.0^{\mathrm{a}}$ & $63.0 \pm 22.0^{\mathrm{b}}$ \\
\hline
\end{tabular}

Values presented as mean \pm SD. For each variable, values followed by different letters are significantly different. SAHS: sleep apnoea/hypopnoea syndrome; BMI: body mass index; AHI: apnoea/hypopnoea index.

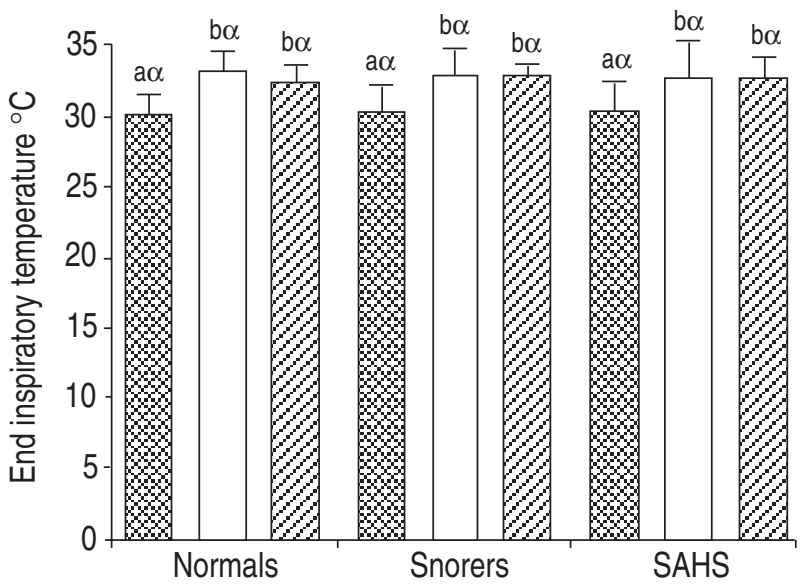

Fig. 2. - End-inspiratory temperature of the different upper airway (UA) regions in each group. The uvular temperature ( $\$$ ) was significantly lower than that of the other UA regions, with no difference between the posterior pharyngeal wall $(\square)$ and the velum ( $\square)$ in the three groups. For a given UA region, there was no difference in UA temperature between the different groups. Data are shown as mean (SD). Columns with different letters are significantly different. Letters refer to the comparison between UA regions within each group of subjects and greek letters are used for comparison between groups for a given UA region. SAHS: sleep apnoea/hypopnoea syndrome.

differed between inspiration and expiration and during the course of the apnoea. For this reason the different UA regions were characterized by their inspiratory temperature, with the inspiratory-expiratory temperature difference used to characterize the expiratory rewarming and by the changes in UA temperature during apnoea. There was no difference in the inspiratory and expiratory time between the different groups. The mean values of the end-inspiratory temperature of the different UA regions in each group are presented in figure 2 . The temperature of the uvula was significantly lower than that of the other UA regions, with mean differences ranging from $0.4-1.5^{\circ} \mathrm{C}$. No difference was found between the posterior pharyngeal wall and the velum temperatures in any group. For each anatomical region, there was no difference between the normals, snorers and SAHS (fig. 2).

The expiratory changes in UA temperature observed in the different UA sites and in each group are given in figure 3 . In normal subjects, the expiratory rewarming was significantly higher in the uvula than in the velum and the posterior pharyngeal wall; however, these regional differences in the inspiratory-expiratory difference in tissue temperature were not observed in snorers and SAHS.
The uvular rewarming was not significantly different between the subjects of the three groups. However, significant differences were observed between the three groups for the inspiratory-expiratory temperature difference at the velum and posterior pharyngeal wall, with the rewarming being significantly larger in snorers and in SAHS than in normals (fig. 3 ).

Since the inspiratory-expiratory temperature differences could be accounted for by the effects of heated exhaled gases on UA, the same measurements were obtained during the course of a voluntary apnoea following a tidal inspiration. Rewarming was quantified by the changes in temperature between the beginning and the end of a $10 \mathrm{~s}$ of apnoea. After exclusive nasal breathing no significant

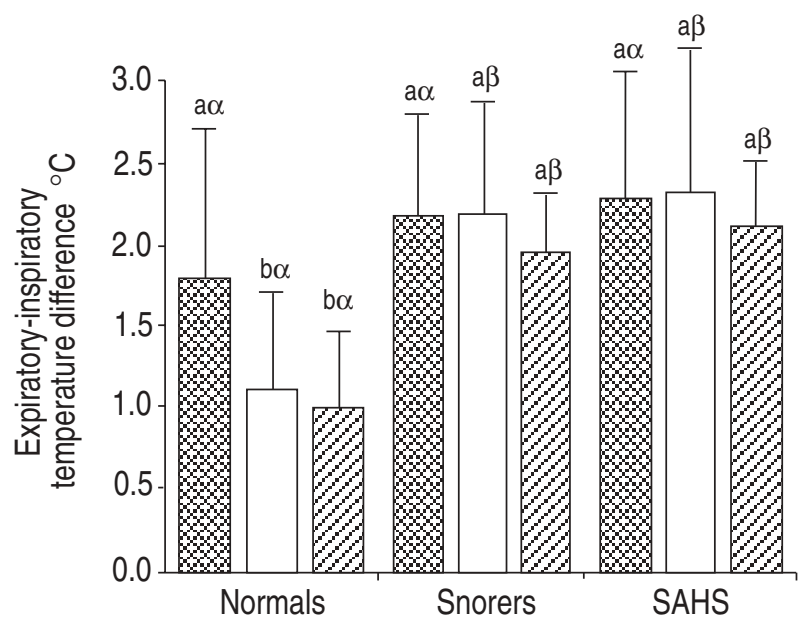

Fig. 3. - Inspiratory-expiratory differences in temperature of the different upper airway regions in each group. In normal subjects, the expiratory increase in uvular ( $\$$ ) temperature was higher than in the post posterior wall ( $\square$ ), and the velum ( $Q$ ). These regional differences were not observed in snorers and subjects with sleep apnoea/hypopnoea syndrome (SAHS). The uvular expiratory-inspiratory temperature difference was not significantly different among the three groups. The velar and posterior pharyngeal expiratory rewarming was significantly higher in snorers and SAHS than in normals. See figure 2 for an explanation of the symbols.

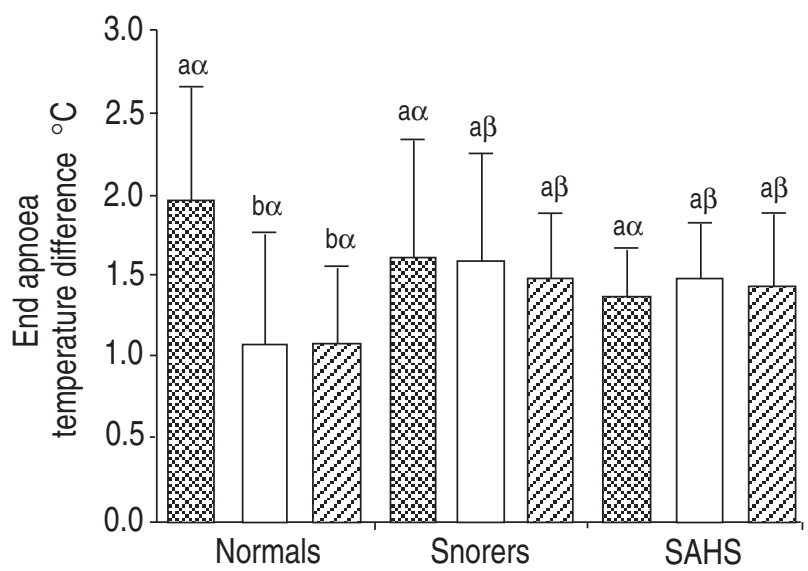

Fig. 4. - Mean change in upper airway (UA) temperature observed during a $10 \mathrm{~s}$ apnoea. As during tidal breathing, the uvular ( $\$$ ) rewarming was significantly higher than that of the other UA sites in normal subjects. For the posterior pharyngeal wall $(\square)$ and the velum $(Z)$ ) this rewarming was higher in snorers and subjects with sleep apnoea/hypopnoea syndrome (SAHS) than in normals. See figure 2 for an explanation of the symbols. 
changes in UA temperature were observed from the beginning to the end of the apnoea. When the apnoea followed exclusive mouth breathing, a rewarming of the UA was observed. As illustrated in figure 4, this rewarming was similar to that observed during expiration. Similar differences were observed between the different UA regions and the different groups, with a significantly higher rewarming in the uvula than in the other regions in normals, no difference in rewarming between the three studied UA sites in snorers and SAHS, and a smaller rewarming in the posterior pharyngeal wall and in the velum in normals than in snorers and SAHS (fig. 4). There was no correlation between the average temperature of the different anatomical sites and nocturnal breathing characteristics (snoring index or apnoea/hypopnoea index).

\section{Discussion}

The results show that tissue temperature varies within the different UA regions during mouth breathing and that the intensity of the regional expiratory and breath-holding rewarming differs among normal subjects, snorers and SAHS.

The observed changes in UA temperature may be accounted for by the balance between the influence of inspiratory and expiratory airstream temperature and of the intrinsic rewarming characteristics of these tissues. These two factors can be evoked to explain the results obtained during tidal breathing. The lower end-inspiratory temperature measured in the uvula than in other pharyngeal regions could be explained by differences in the aerodynamic pattern within the UA that would influence the temperature exchange between inhaled gas and UA tissues and/or by differences in the local blood flow. Since the regional inspiratory cooling was similar in the three groups, even if group differences were observed in the expiratory rewarming pattern, inspiratory changes in UA temperature seem to be determined mainly by the effects of inspired air temperature. Exhalation of heated air may contribute to UA rewarming; however, other factors may contribute to explain the differences in the intensity of the rewarming observed between the three groups, such as differences in the intrinsic temperature of these tissues, e.g. as a consequence of differences in local blood flow. During apnoea, the UA rewarming can only be accounted for by intrinsic tissue properties. The similarity between the results obtained at end-expiration and at the end of an apnoea suggests that this factor contributes primarily in determining the intensity of rewarming in both conditions. Therefore, the observed increase in the end-expiratory and/or endapnoeic temperature rewarming may be consequential to UA inflammation, as suggested for gingival mucosa, the rewarming rate of which has been shown to increase with increasing intensity of tissue inflammation [11]. No technical bias could contribute to explain the similarity of the uvular rewarming rate among the three groups while the velar and posterior pharyngeal rewarming differed significantly between normals and the two other groups. One possible explanation could be that local mucosal fat deposition may have interfered with changes in tissue temperature [16].

There is indirect evidence that sleep-related breathing disorders induce UA inflammation in SAHS. Using mag- netic resonance imaging (MRI), RYAn et al. [17] found a decrease in the mucosal water content following continuous positive airway pressure (CPAP) therapy in these patients. This decrease occurred mainly at the oropharyngeal level and was accompanied by a decrease in the volume of both the tongue and soft palate and an increase in pharyngeal cross-sectional area. These findings were attributed to the relief of UA oedema by CPAP therapy. Similar findings have been reported recently by MoRTIMORE et al. [18] who used cephalometric measurements to show that CPAP therapy caused a significant increase in the posterior airway space. The beneficial effects of CPAP on UA oedema suggest that the oedema is linked with the mechanical consequences of UA collapse, i.e. possibly as a consequence of the relief of vascular and lymphatic damming. The differences observed between SAHS and normals are consistent with such consequences of UA trauma. Interestingly, the similarity of the results obtained in snorers and SAHS and the differences observed between the snorers' groups and normal nonsnorers suggest that UA oedema is as important in snorers as in SAHS. This is consistent with the findings of Woobson et al. [6] who found no qualitative differences in histopathological characteristics between SAHS and nonapnoeic snorers. Therefore, UA fluttering and UA collapse may have similar consequences on UA tissue characteristics. In both groups, UA oedema may by itself contribute to worsen breathing abnormalities, as illustrated by the increase in UA collapsibility as-sociated with experimentally induced vasodilatation [19] and the decrease in UA collapsibility observed in SAHS following CPAP therapy [20]. This is consistent with the decrease in the apnoea+hypopnoea index that is observed on the first night following withdrawal of regular CPAP therapy [21]. The same observation was made in nonapnoeic snorers, whose snoring severity significantly impro-ved immediately following the interruption of 30 days of CPAP therapy [22]. Since significant differences were observed in soft tissue expiratory rewarming between ap-noeic and nonapnoeic subjects, the diagnostic value of the inspiratory-expiratory temperature difference at the velum and the posterior pharyngeal wall in identifying apnoeic patients was assessed. Using a $2.3^{\circ} \mathrm{C}$ temperature difference for the normal threshold (mean+ 2 SD of normal values) at the posterior pharyngeal wall, the sensitivity was $60 \%$, specificity $87.5 \%$, positive predictive value $66.7 \%$ and negative predictive value $84 \%$. For velar temperature differences using threshold of $1.8^{\circ} \mathrm{C}$, the sensitivity was $70 \%$, specificity $70.8 \%$, positive predictive value $50 \%$ and negative predictive value $85 \%$.

To the authors' knowledge, only a few histological studies have been conducted to investigate the presence of inflammatory process in UA tissues of SAHS and snorers. A recent study has found that the nasal lavage composition significantly differs between SAHS and normal subjects, with an increased proportion of polymorphonuclear leukocytes and pro-inflammatory mediators in the former group [23]. This demonstrates that tissue inflammation is present in the most proximal end of UA, but the nasal lavage procedure does not allow a precise localization of the inflammatory site(s) inside the nasopharyngeal airways. The present findings suggest that this inflammatory process may not be limited to the nasopharynx but may also involve the oropharyngeal airway. 
Interestingly, preliminary results from the same laboratory suggest that the catabolism of pro-inflammatory mediators may be altered in the uvula of SAHS patients [24].

The infra-red recording system used in this study allowed only perioral measurements of UA tissue temperature to be obtained. Therefore, posterior velar and hypopharyngeal regions could not be explored in the present study. Furthermore, since infra-red waves are not transmitted through optic fibres, these measurements cannot be made through conventional endoscopes. However, the ongoing developments in the field of infra-red spectroscopy could allow such analysis in the future [25] and this would be very useful in mapping the temperature of tissues and localizing inflammatory structures during any endoscopic procedure. In SAHS, this could be helpful to identify easily the anatomical regions involved in UA closure and potentially be used to identify good responders to velopharyngeal surgery.

In conclusion, significant changes occur in the temperature of certain pharyngeal regions of snorers and obstructive sleep apnoea patients, in contrast to normal controls. These differences can be accounted for by mucosal upper airway properties and particularly by the presence of upper airway inflammation. Further studies will be required to determine the importance of this finding with respect to the possibility of localizing sites of upper airway collapse.

Acknowledgement: The authors would like to thank S. Simard for statistical analysis.

\section{References}

1. Morisson DL, Launois SH, Isono S, Feroah TR, Whitelaw WA, Remmers JE. Pharyngeal narrowing and closing pressures in patients with obstructive sleep apnea. Am Rev Respir Dis 1993; 148: 606-611.

2. Gleadhill IC, Schwartz AR, Schubert N, Wise RA, Permutt S, Smith PL. Upper airway collapsibility in snorers and in patients with obstructive hypopnea and apnea. Am Rev Respir Dis 1991; 143: 1300-1303.

3. Liistro G, Stãnescu DC, Veriter C, Rodenstein DO, Aubert-Tulkens G. Pattern of snoring in obstructive sleep apnea patients and in heavy snorers. Sleep 1991; 14: 517525.

4. Remmers JE, DeGroot WJ, Sauerland EK, Anch AM. Pathogenesis of upper airway occlusion during sleep. $J$ Appl Physiol 1978; 44: 931-938.

5. Wilson SL, Thach BT, Brouillette RT, Abu-Osba Y. Upper airway patency in the human infant: influence of airway pressure and posture. J Appl Physiol 1980; 48: 500-504.

6. Woodson BT, Garancis JC, Toohill RJ. Histopathologic changes in snoring and obstructive sleep apnea syndrome. Laryngoscope 1991; 101: 1318-1322.

7. Chan AW, McFarlane IA, Bowsher DR. Contact thermography of painful diabetic neuropathic foot. Diabetes Care 1991; 14: 918-922.

8. Bothmann G. Liquid crystal thermography of the breast.
Eur J Gynaecol Oncol 1986; 7: 88-92.

9. McDonald AG, Land DV, Sturrock RD. Microwave thermography as a noninvasive assessment of disease activity in inflammatory arthritis. Clin Rheumatol 1994; 13: 589592.

10. White BA, Lockhart PB, Connolly SF, Sonis ST. The use of thermography in the evaluation of oral lesions. Int $J$ Tissue React 1987; 9: 105-114.

11. Barnett ML, Gilman RM, Charles CH, Bartels LL. Computer-based thermal imaging of human gingiva: preliminary investigation. J Periodontol 1989; 60: 628-633.

12. Sériès F, Sériès I, Atton L. Comparison of snoring characteristics obtained by polysomnographic studies and home recordings. Chest 1993; 103: 1769-1773.

13. Rechtschaffen A, Kales A. A manual of standardised terminology, techniques and snoring system for sleep stages of human subjects. Washington DC, Public Health Service, National Institutes of Health, 1968; publication 204.

14. American Sleep Disorders Association. EEG arousals: scoring rules and examples. Sleep 1992; 15: 174-183.

15. Currie NC, Demma EJ, Ferris DD, Kwasowsky BR, McMillan RW, Wicks MC. Infrared and millimeter-wave sensors for military special operations and law enforcement applications. Int J Infrared Milli 1996; 17: 1117-1138.

16. Stauffer JL, Buick MK, Bixler EO, et al. Morphology of the uvula in obstructive sleep apnea. Am Rev Respir Dis 1989; 140: 724-728.

17. Ryan CF, Lowe AA, Li D, Fleetham JA. Magnetic resonance imaging of the upper airway in obstructive sleep apnea before and after chronic nasal continuous positive airway pressure therapy. Am Rev Respir Dis 1991; 144: 939-944.

18. Mortimore IL, Kochhnar P, Douglas NJ. Effect of chronic continuous positive airway pressure (CPAP) therapy on upper airway size in patients with sleep apnoea/hypopnoea syndrome. Thorax 1996; 51: 190-192.

19. Wasicko MJ, Hutt DA, Parisi RA, Neubaeur JA, Mezrich $\mathrm{R}$, Edelman NH. The role of vascular tone in the control of upper airway collapsibility. Am Rev Respir Dis 1990; 141: 1569-1577.

20. Smith PL, Gold AR, Meyers DA, Haponick EK, Bleecker ER. Weight loss in midly to moderately obese patients with obstructive sleep apnea. Ann Intern Med 1985; 103: 850-855.

21. Kribbs NB, Pack AI, Kline LR, et al. Effects of one night without nasal CPAP treatment on sleep and sleepiness in patients with obstructive sleep apnea. Am Rev Respir Dis 1993; 147: 1162-1168.

22. Sériès F, Marc I. Changes in snoring characteristics after 30 days of nasal continuous positive airway pressure in patients with non-apnoeic snoring: a controlled trial. Tho$\operatorname{rax} 1$ 1994; 49: 562-566

23. Rubinstein I. Nasal inflammation in patients with obstructive sleep apnea. Laryngoscope 1995; 105: 175-177.

24. Zakkar M, Sekosan M, Wenig B, Olopade CO, Rubinstein I. Decrease in immunoreactive neutral endopeptidase in uvula epithelium of patients with obstructive sleep apnea. Am J Respir Crit Care Med 1996; 153: A692.

25. Lin J, Brown CW. Near-IR fiber-optic temperature sensor. Appl Spectrosc 1993; 47: 62-68. 